\title{
Ferramenta de Simulação com Abordagem de Sistema Multiagente Híbrida para Gestão Ambiental
}

\author{
Carolina Gonçalves Abreu ${ }^{1,2}$, Cássio Giorgio Couto Coelho ${ }^{2}$, \\ Célia Ghedini Ralha ${ }^{2}$, Alexandre Zaghetto ${ }^{2}$, Bruno Macchiavello ${ }^{2}$ \\ ${ }^{1}$ Instituto Brasileiro do Meio Ambiente e dos Recursos Naturais Renováveis (IBAMA) \\ SCEN Trecho 2 - Ed. Sede - Cx. Postal no 09566 - CEP 70818-900 - Brasília-DF \\ carolina.abreu@ibama.gov.br \\ ${ }^{2}$ Departamento de Ciência da Computação - Universidade de Brasília (UnB) \\ Caixa Postal 4466 - 70.904-970 - Brasília - DF - Brasil \\ \{cassiocouto, ghedini, alexandre, bruno\}@cic.unb.br
}

\begin{abstract}
This paper introduces a prototype for a computer system that performs simulations of land use. The proposed system is an evolution of an earlier work and aims to assist in analyzing the dynamics of land use and land cover, so it can serve as a tool in making decisions. The system uses a multi-agent approach and configurable model. The model takes into account certain proximal variables such as the presence of roads, buildings, water courses, etc. These variables are used in order to identify which regions are most likely to be used in any anthropic activity. The system presents the potential for the user to be able to configure the simulation model indicating which proximal variables will be taken into account, the importance of each variable, and even the state machine used during the simulations. The multi-agent system allows the development of various behaviors of the agents involved which can generate different simulation's scenarios. Initial simulations are also presented for the cerrado biome. The cerrado is one of the major biomes and is considered a hotspot of global biodiversity.
\end{abstract}

Resumo. O presente artigo introduz um protótipo para um sistema computacional que possibilita a realização de simulações do uso do solo. O sistema proposto é uma evolução de um trabalho anterior e tem como objetivo auxiliar na análise da dinâmica do uso e cobertura do solo, de tal forma que possa servir como uma ferramenta utilizada na tomada de decisões. O sistema utiliza uma abordagem multiagentes e modelo configurável pelo usuário. O modelo leva em consideração certas variáveis proximais como por exemplo a presença de estradas, edificações, cursos de água, etc. Estas variáveis proximais são utilizadas para identificar as regiões com maior probabilidade de uso antrópico. O sistema apresenta o potencial para que o usuário seja capaz de configurar o modelo de simulação indicando quais variáveis de proximais serão levadas em consideração, a importância de cada uma delas e inclusive a maquina de estados a ser utilizada durante a simulação. O sistema multiagentes permite a elaboração de diversos comportamentos dos agentes envolvidos podendo gerar diferentes cenários de simulação. São também apresentadas simulações iniciais para o bioma do cerrado. O cerrado é um dos maiores biomas brasileiros e é considerado um hotspot da biodiversidade mundial. 


\section{Introdução}

Têm-se observado que os sistemas de tomada de decisão tendem a separar os fatores ambientais do plano político e de planejamento, não incluindo-os de maneira significativa seja na formulação de políticas públicas ou na resolução de questões cruciais de gestão ambiental. Dessa maneira, uma miríade de informações ambientais que são produzidas por atores técnicos não tem o seu potencial explorado para embasar a tomada de decisão. Um exemplo claro desse distanciamento entre a informação e a ação é o Bioma Cerrado.

O Cerrado é o segundo maior bioma brasileiro, ocupando $21 \%$ do território nacional com uma das mais ricas savanas do mundo. É considerado um hotspot da biodiversidade mundial, requerendo ações urgentes para a conservação pelo seu alto nível de endemismo e avançado grau de perda de habitat. Entretanto, são altíssimas as taxas de desmatamento nesse bioma, muito superiores inclusive às taxas de desmatamento na Floresta Amazônica. Os dados do Instituto Brasileiro do Meio Ambiente e dos Recursos Naturais Renováveis - Ibama, mostram uma perda acumulada de 47,8\% de cobertura vegetal natural do Cerrado até 2008. Especialistas apontam que existe um esforço de conservação muito inferior às reais necessidades do bioma. Somente $2,2 \%$ do território ocupado pelo Cerrado é legalmente protegido [Klink and Machado 2005].

Essa rápida e intensa exploração se dá principalmente pelas mudanças na cobertura e uso do solo, principalmente porque o Cerrado é considerado uma das últimas fronteiras de produção agrícola do mundo. As mudanças no uso e cobertura do solo são relevantes não somente para este bioma, pois são consideradas uma das principais fontes de alteração recente na superfície terrestre. Portanto, o entendimento da dinâmica desses fenômenos é condição precípua para uma adequada política de uso sustentável dos recursos.

Uma das ferramentas que podem ser aplicadas para entender essa dinâmica são os modelos, em especial os modelos de simulação. As simulações são vistas como instrumentos de aquisição do conhecimento e de apoio à decisão [Verburg 2006]. Pela tentativa de reproduzir os mecanismos da realidade, esses modelos podem mostrar como as escolhas de como o solo é usado afetam as direções futuras desse sistema. Na área da Ciência da Computação, a subárea da Inteligência Artificial (IA), com ênfase na abordagem de Sistemas Multiagentes (SMA) [Wooldridge 2009] surge como técnica candidata à resolução de problemas de modelos de simulação dinâmica. A abordagem de SMA lida com a autonomia dos agentes ganhando representatividade para fins de organização de sistemas constituídos por interações entre os diversos agentes de forma complexa e inteligente [Weiss 2000]. A relação entre os processos biofísicos e socioeconômicos pode ser representada pela dinâmica entre agentes heterogêneos que interagem entre si e com o ambiente.

A partir desse contexto, o problema é entender a dinâmica do uso e cobertura do solo no Cerrado para contribuir na investigação de cenários mais favoráveis para a manutenção da cobertura vegetal natural, visando o equilíbrio entre políticas de conservação e o avanço das atividades de uso do solo para exploração econômica. O sistema proposto é uma evolução de um trabalho anterior [Marinelli et al. 2010] e visa desenvolver uma ferramenta computacional de simulação, baseada na abordagem de SMA, para analisar a dinâmica de uso e cobertura do solo, utilizando como estudo de caso o bioma Cerrado. 


\section{Comparação entre Ferramentas}

Existem várias ferramentas descritas na literatura que empregam perspectivas e técnicas distintas para abordar o problema da simulação dinâmica das mudanças no uso do solo. Foram comparadas as tecnologias e funcionalidades de algumas dessas ferramentas, além da complexidade dos modelos, utilizando o framework descrito em trabalhos anteriores [Agarwal et al. 2001], onde são analisados parâmetros de complexidade de espaço, tempo e tomada de decisão humana. Algumas ferramentas foram selecionadas para exemplificar a variedade de soluções hoje existentes, com suas características apresentadas na Tabela 1.

O CORMAS (Common-Pool Resources and Multi-agent Systems) [Bousquet et al. 1998], desenvolvido pela French Agricultural Research Centre for International Development (CIRAD), é uma ferramenta de simulação que utiliza abordagem SMA, construída na plataforma Visual Works com a linguagem Smaltalk. Foi projetada para ser um ambiente de fácil desenvolvimento, dedicado à modelagem das interações das sociedades humanas com os recursos renováveis. Entretanto, a abordagem de SMA utilizada não faz uso de racionalidade nos agentes. O trabalho anterior do grupo utilizou a ferramenta CORMAS para realizar uma simulação em uma reserva de desenvolvimento sustentável na Amazônia [Marinelli et al. 2010].

A Universidade Federal de Minas Gerais (UFMG) destaca-se com a ferramenta de domínio público Dinamica-EGO [Soares-Filho et al. 2002]. O Dinamica-EGO é um simulador com cálculo estocástico, baseado em formalismo de autômato celular, para replicação dos padrões espaciais de mudanças da dinâmica de paisagens. O DinâmicaEGO possibilita a concepção e a aplicação de modelos de simulação para a gestão ambiental. Entretanto, a ferramenta não utiliza técnicas de IA ou SMA.

Modelos de simulação espacial baseados em autômatos celulares têm se tornado populares, sendo incluídos em softwares comerciais de Sistemas de Informações Geográficas, como o IDRISI [Eastman 2001], devido à sua facilidade de implementação, habilidade em mimetizar formas e capacidade de serem readaptados para produzir vários tipos de fenômenos espaciais. IDRISI utiliza a combinação de autômato celular com modelo estocástico. Existe um módulo projetado para as mudanças no uso do solo, o LCM - Land Change Modeler, mas que não utiliza técnicas de SMA. Aplica somente técnicas estatísticas para avaliar a mudança no espaço, tendo por base a imagem em dois tempos distintos.

Em [Verburg et al. 2002] é apresentado o framework de modelagem CLUE - The Conversion of Land Use and its Effects. É uma plataforma específica para as investigações de uso e cobertura do solo, por meio de um modelo discreto cujas funções de mudança são baseadas em cálculos estatísticos. Possui uma limitação espacial, sendo mais adequado para aplicação em pequenas regiões, e não incorpora técnicas de SMA.

Algumas críticas em relação às ferramentas estão descritas em vários artigos de revisão. Um ponto presente em quase todos os trabalhos é a questão das escalas. Nenhuma ferramenta pesquisada permite análises em múltiplas escalas simultaneamente. Outra crítica diz respeito ao modo como as ferramentas interagem no espaço, ao considerarem o espaço homogêneo, ao aplicarem regras de transição universais ou ao permitirem unicamente vizinhanças estácionárias. Especificamente para as ferramentas de abordagem SMA, muito questiona-se acerca da fragilidade da racionalidade dos agen- 
Tabela 1. Características das ferramentas

\begin{tabular}{|c|c|c|c|c|}
\hline Características & Dinamina-EGO & IDRISI (LCM) & $\overline{\text { CLUE }}$ & CORMAS \\
\hline $\begin{array}{l}\text { Tipo de } \\
\text { Modelo }\end{array}$ & $\begin{array}{l}\text { Modelos espaciais } \\
\text { dinâmicos }\end{array}$ & $\begin{array}{c}\text { Modelo espacial } \\
\text { de Markov }\end{array}$ & $\begin{array}{l}\text { Modelo de estado } \\
\text { discreto finito }\end{array}$ & $\begin{array}{l}\text { Simulações } \\
\text { Multiagentes }\end{array}$ \\
\hline Tecnologia & Autômato Celular & Autômato Celular & Matrizes de conversão & Sistema Multiagente \\
\hline Hierarquia & top-down & top-down & top-down & bottom-up \\
\hline $\begin{array}{l}\text { Complexidade } \\
\text { Espacial }\end{array}$ & $\begin{array}{l}\text { Representação e } \\
\text { interação }\end{array}$ & Representação & $\begin{array}{c}\text { Representação e } \\
\text { interação }\end{array}$ & $\begin{array}{l}\text { Representação e } \\
\text { interação }\end{array}$ \\
\hline Escala & Raster & Raster & Raster & Raster \\
\hline Resolução & Matriz Quadrada & Definida pelo usuário & Definida pelo usuário & Definida pelo usuário \\
\hline $\begin{array}{c}\text { Tomada de } \\
\text { Decisão Humana }\end{array}$ & $\begin{array}{l}\text { Assumida como relacionada } \\
\text { ao tamanho da população, } \\
\text { densidade, etc. }\end{array}$ & $\begin{array}{l}\text { Nenhuma, só variáveis } \\
\text { biofísicas no modelo }\end{array}$ & $\begin{array}{l}\text { Função de probabilidade } \\
\text { que depende de variáveis } \\
\text { socioeconômicas }\end{array}$ & $\begin{array}{c}\text { Variável conforme o } \\
\text { comportamento } \\
\text { dos agentes }\end{array}$ \\
\hline
\end{tabular}

tes. Alguns apontamentos de ordem mais geral observam que é necessário conferir maior credibilidade aos modelos, por meio de verificações e validações consistentes. As características técnicas de cada uma das ferramentas, bem como as críticas foram consideradas no projeto da ferramenta de simulação aqui apresentada.

\section{Apresentação dos Modelos}

O modelo definido representa a cobertura e o uso do solo do Distrito Federal, unidade federativa que tem $100 \%$ do seu território na região do Cerrado. Com área de 5.789, $16 \mathrm{Km}^{2}$, estima-se que $68,11 \%$ da vegetação nativa já foi desmatada. Foram utilizadas as imagens do Projeto de Monitoramento do Desmatamento dos Biomas Brasileiros por satélite (Ministério do Meio Ambiente - MMA/Ibama) e o Mapa de Cobertura Vegetal dos Biomas Brasileiros (MMA), escala 1:250.000, ano base 2002.

\subsection{Classes de Uso do Solo}

Para a definição das classes a serem utilizadas no modelo, trabalhos recentes de classificação do solo foram consultados. Conforme o mapeamento de Sano [Sano et al. 2008], o DF apresenta as seguintes classes de uso de solo: cultura agrícola, pastagem cultivada, massa d'água, vegetação nativa, e área urbana. Na escala de estudo não foram detectadas áreas de mineração e as áreas de reflorestamento são pouco significativas. A proporção de ocupação das áreas é apresentada na Figura 1.

De acordo com a revisão de sucessão da fronteira agrícola no Cerrado e com o apoio de especialistas, especificou-se uma máquina de estados simples e determinística para o estado de conservação dos solos (Figura 2), com as classes agricultura, pecuária, urbanização, conservação e vegetação nativa. É possível explorar cenários diversos pela alteração das taxas de conversão entre os estados, caracterizando uma perspectiva tendencial (business as usual), conservativa ou degradativa.

\subsection{Variáveis Proximais}

A exploração do uso do solo depende de diversos fatores sociais, econômicos, geográficos e inclusive de caráter político. Muitos destes fatores são difíceis de serem previstos e modelados em um sistema de simulação. Porém, certas informações do ambiente podem ser levadas em consideração para criar um ambiente de simulação do uso do solo. Estas 


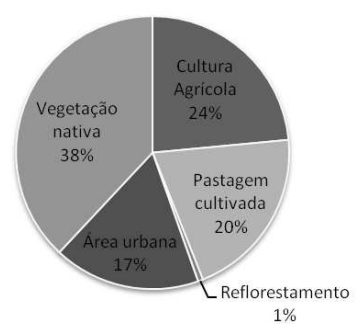

Figura 1. Proporção de áreas ocupadas pelas diferentes classes de uso da terra

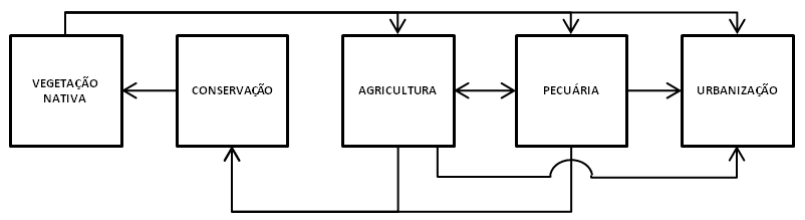

Figura 2. Máquina de estados do modelo

informações são comumente chamadas de variáveis proximais. As variáveis proximais consideradas no presente trabalho foram: Ferrovia, Rodovia, Rio, Lago, Rua, Edificação e Unidade de Conservação. Consideramos que Ferrovia, Rodovia, Rio, Lago, Rua e Edificação estimulam o uso antrópico de áreas situadas em suas imediações. Ou seja, áreas próximas a uma rodovia, por exemplo, são mais propensas a serem ocupadas que áreas mais distantes.

Para se modelar a capacidade de estímulo à ocupação de cada uma dessas variáveis, as imagens que as representam foram filtradas por meio de um filtro Gaussiano isotrópico (circularmente simétrico) bidimensional $h(x, y)$, definido pela Equação 1,

$$
h(x, y)=\frac{1}{2 \pi \sigma^{2}} e^{-\frac{x^{2}+y^{2}}{2 \sigma^{2}}},
$$

onde $h_{\text {size }}$ e $\sigma$ representam o tamanho do filtro e o desvio padrão, respectivamente, e são parâmetros que podem ser ajustados pelo usuário do sistema. A Figura 3 mostra o filtro Gaussiano utilizado na geração das imagens da Figura $4\left(h_{\text {size }}=11\right.$ e $\left.\sigma=2\right)$. A Figura 5 mostra uma parte ampliada para uma variável proximal. As regiões mais claras nas imagens representam os lugares onde existe uma maior probabilidade de ocupação da terra para uso antrópico. As áreas mais escuras, que estão circundadas por pixels mais claros, indicam regiões que não podem ser utilizadas durante o processo de simulação. Estas regiões mais escuras representam áreas que já estão ocupadas, por exemplo por estradas, edificações, etc., ou áreas de curso de água. A variável Unidade de Conservação, mostrada na Figura 6, tem o efeito de inibir o uso antrópico. Nesse exemplo, a região em branco é uma área protegida e não pode ser ocupada.

Após terem sido geradas, as imagens filtradas são utilizadas no cômputo de uma média ponderada, de acordo com a Equação 2,

$$
T=\sum_{i=0}^{5} M_{i} \cdot p_{i}
$$




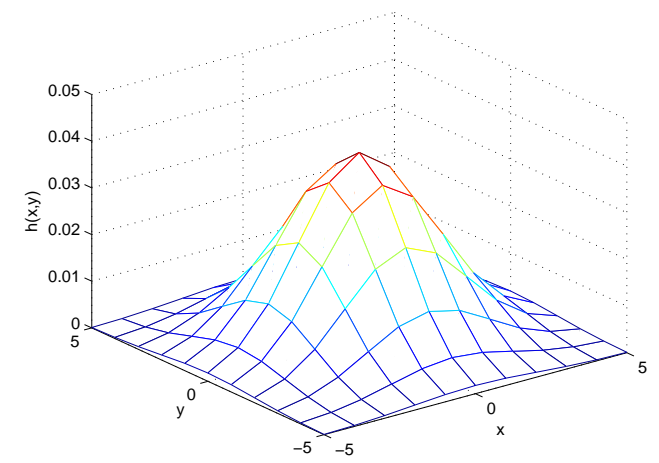

Figura 3. Filtro Gaussiano utilizado na geração das imagens mostradas nas Figuras 4 e 5 .

onde $T$ é o mapa resultante, $M_{i}$ representa a imagem filtrada referente a variável proximal $i$ e $p_{i}$ indica quanto a variável $i$ vai contribuir na geração do mapa $T$. Além disso, gerouse um mapa auxiliar $T_{a u x}$ que indica as regiões desabilitadas para qualquer tipo de uso, por constituirem Unidades de Conservação ou por serem áreas que já estão ocupadas. A Figura 7 mostra os mapas $T$ e $T_{\text {aux }}$ para o cerrado. Todos os pesos $p_{i}$ foram considerados iguais a $1 / 6$.

A Figura 8 mostra uma exemplo de como os diferentes mapas podem ser utilizados para determinar a movimentação de um agente explorador. Tomando como referência a ilustração da Figura 8(a), considere que áreas em amarelo são áreas que já foram ou estão sendo exploradas, e áreas em verde são áreas que potencialmente poderiam ser exploradas. O círculo indica um agente explorador. Uma vez que haja a necessidade de movimentação do agente, o mapa $T_{a u x}$, mostrado na Figura 8 (b), é consultado e as células vizinhas marcadas com o valor lógico 1 são identificadas com potenciais distinos. Note que nesse exemplo estão sendo descartadas as células que já foram exploradas (em amarelo na Figura 8(a)) ou que estão marcadas com valor lógico 0. Em seguida o mapa $T$ é consultado para que dentre as células candidatas a destinos seja escolhida aquela que apresenta o maior potencial de atração do agente explorador. Essa célula, então, passa a ser a nova área em exploração.

\section{Protótipo}

O protótipo da ferramenta implementa o modelo descrito na seção anterior, configurando as mudanças ocorridas no tempo por meio das variáveis proximais definidas no modelo, bem como pela racionalidade definida para cada um dos agentes. Esta proposta de solução considera camadas (layers) arquiteturais para a simulação.

A ferramenta proposta pode ser sintetizada como um SMA híbrido de coordenação competitiva. SMA por ser composto por vários agentes, os quais perseguem um objetivo de projeto. A abordagem de interação entre os agentes pode ser definida como competitiva, uma vez que os agentes do uso do solo concorrem pelo mesmo recurso (o espaço para uso do solo) dentro do grid de simulação e podem ter objetivos distintos e concorrentes. A abordagem híbrida se refere à comunicação e interação dos agentes, permitindo, uma abordagem multiescalar, como mostrado na Figura 9. 


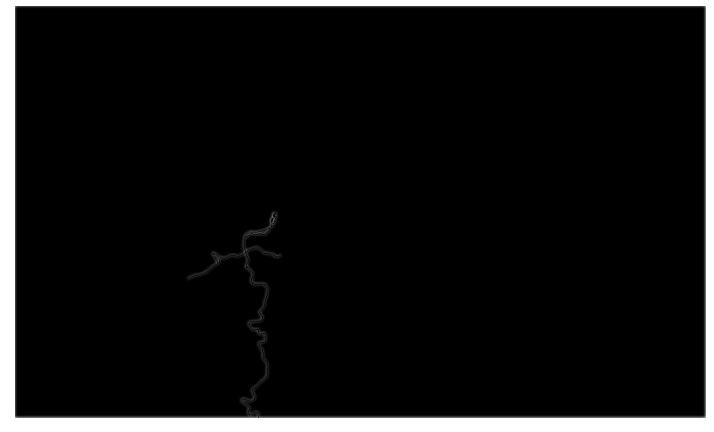

(a) Ferrovia

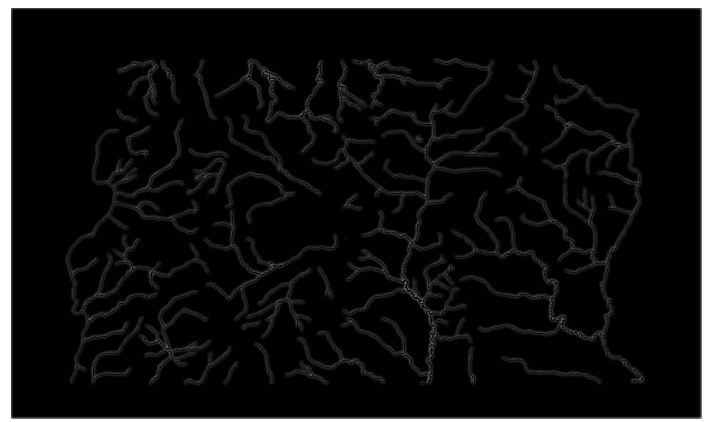

(c) Rio

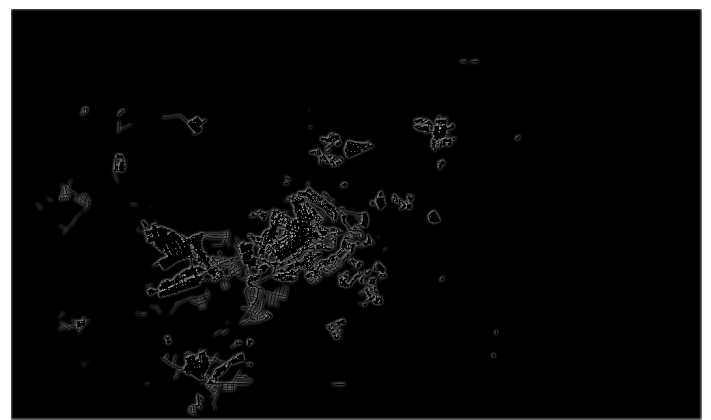

(e) Rua

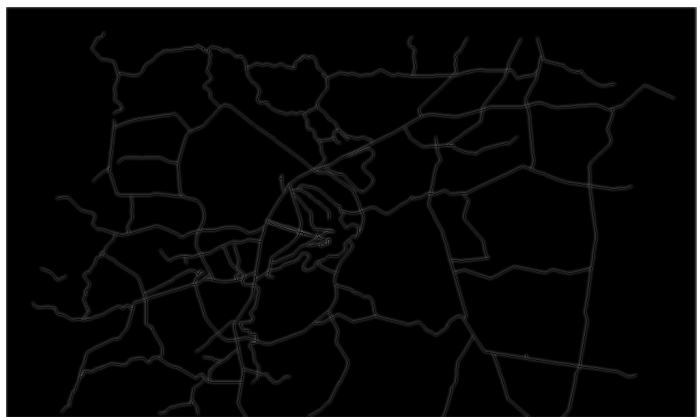

(b) Rodovia

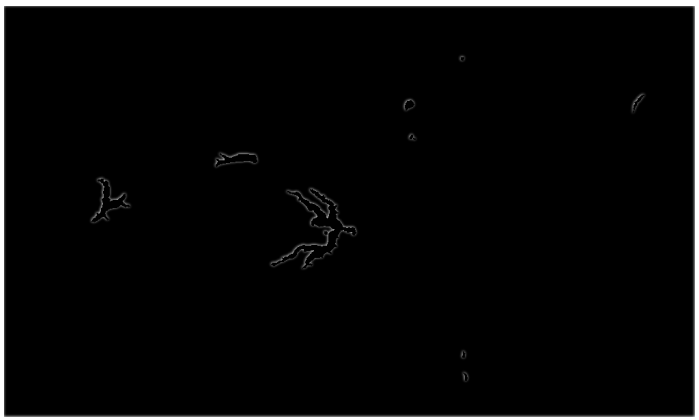

(d) Lago

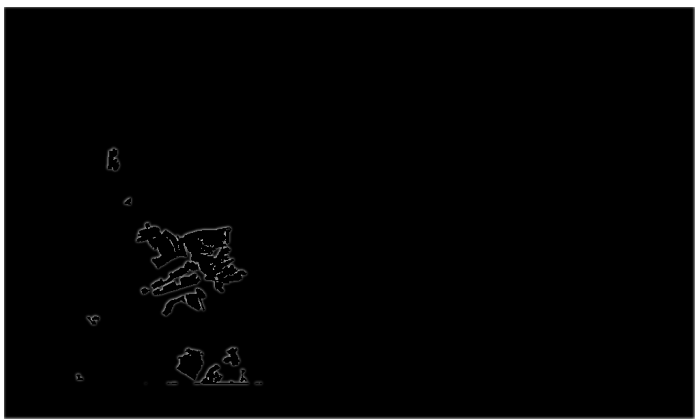

(f) Edificação

Figura 4. Variáveis proximais.

A estrutura top-down para o modelo de uso do solo assume que a taxa de mudança pode ser determinada para a região como um todo. Na estrutura bottom-up, típica dos SMA tradicionais, a análise ocorre em cada pixel, sendo a mudança resultante de todas as decisões locais. A proposta híbrida conjuga as duas hierarquias, permitindo maior flexibilidade de análise.

\subsection{Framework de Desenvolvimento}

O desenvolvimento do protótipo utiliza o Java Agent Development Framework (JADE), que implementa a arquitetura proposta pela FIPA (Foundation for Intelligent Physical Agents) para definição de um SMA. A FIPA é uma organização pertencente a instituição IEEE (Institute of Electrical and Electronics Engineers) que especifica padrões para o desenvolvimento de tecnologias baseadas em agentes inteligentes. O framework JADE, desenvolvido pela TILAB (Telecom Italia $L A B$ ) é um middleware para desenvolvimento 


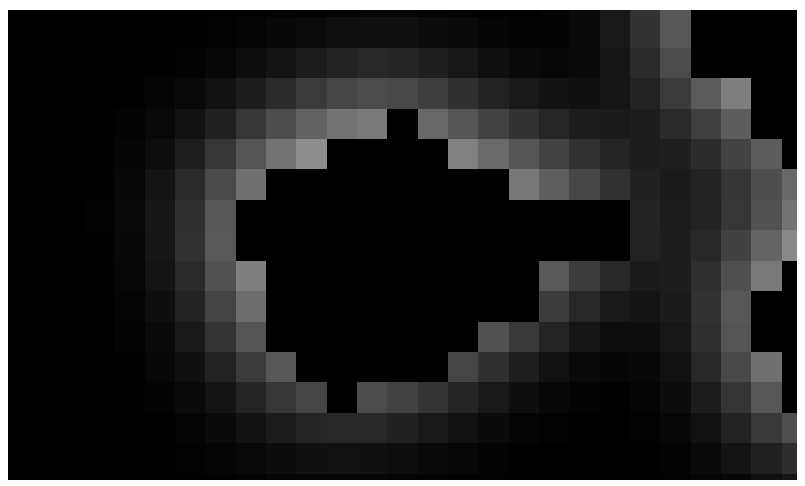

Figura 5. Parte ampliada da variável proximal Edificações. Segundo o que está sendo proposto, regiões mais claras estariam mais propensas a ocupação que regiões mais escuras.

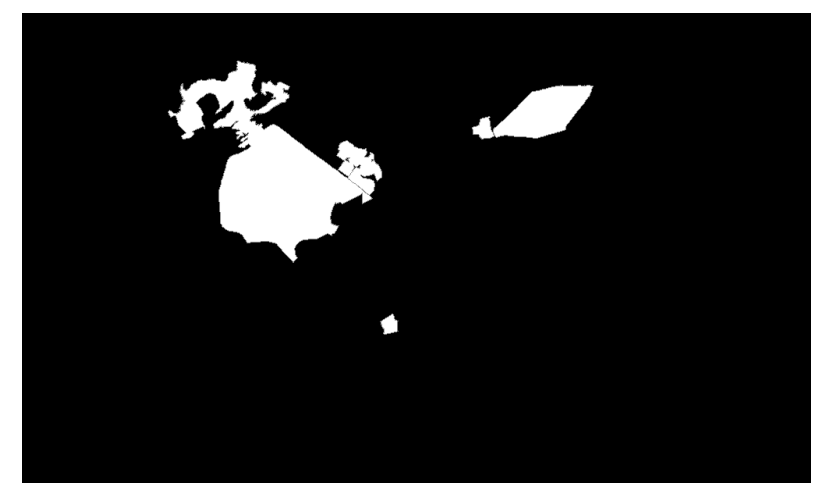

Figura 6. Unidade de Conservação: inibe o uso antrópico.

e execução de aplicações baseadas em agentes inteligentes, desenvolvido na linguagem Java. Fornece serviços de comunicação e interação de agentes, além de ferramentas para a mobilidade de agentes entre plataformas [Bellifemine et al. 2008].

Para a racionalidade dos agentes utiliza-se o DROOLS, um motor de inferência para construção de bases de conhecimento e inferência dirigida por padrão nos agentes inteligentes. O DROOLS é feito para interagir com Java usando conhecimento vindo das regras declarativas nele implementadas. Um dos aspectos relevantes é que o DROOLS fornece uma sintaxe declarativa e é bastante flexível para combinar a semântica do domínio do problema com a linguagem de programação. Tais características permitem, por exemplo, definir regras para a sucessão de classes de uso do solo.

Para os cálculos dos mapas de influência, foi utilizado a ferramenta simulink do software MATLAB, exportado pela biblioteca matlabcontrol, que provê a interface e permite que agentes JADE interajam com sistemas desenvolvidos em MATLAB. Ademais, optou-se pela utilização de XML para configuração do modelo a ser simulado e pela persistência de dados em MySQL com framework Hibernate.

\subsection{Proposta Inicial de Arquitetura}

Foram propostas classes de agentes, delimitando entidades responsáveis pelas tomadas de decisão, pela execução das ações, pelo ambiente de visualização e pela simulação. Até o momento foram criadas cinco classes de agentes conforme apresentado na Tabela 2, 


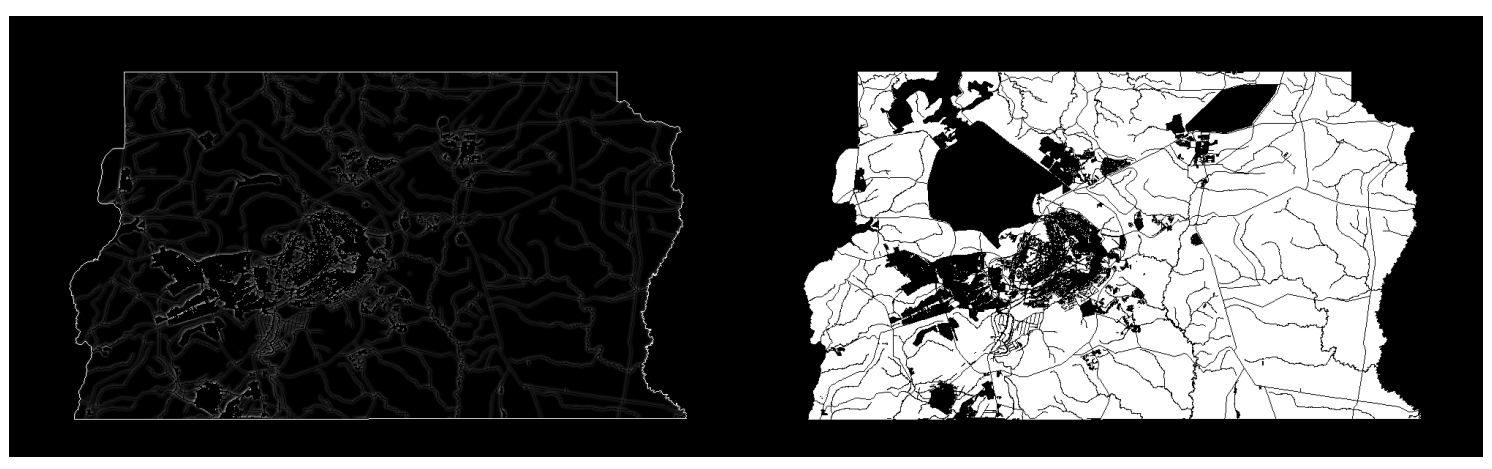

(a) Mapa $T$

(b) Mapa $T_{a u x}$

Figura 7. Mapas $T$ e $T_{a u x}$ resultantes, utilizados como indicadores de propensão à ocupação. Em (a), regiões mais clara indicam maior propensão à exploração. Em (b) regiões mais escuras indicam impossibilidade de exploração.

Tabela 2. Descrição das Classes de Agentes.

\begin{tabular}{l|l|l|l}
\hline \hline Agentes & Tipo & Qdade Instâncias) & Funções \\
\hline \hline $\begin{array}{l}\text { Gerente de } \\
\text { GRID (GGRID) }\end{array}$ & $\begin{array}{l}\text { Orientado } \\
\text { a Objetivos }\end{array}$ & 1 & $\begin{array}{l}\text { Promover interface dos comandos do usuário } \\
\text { Ordenar início, pausa, fim dos agentes } \\
\text { Recebe estados dos agentes para visualização } \\
\text { Promove a visualização dos estados dos agentes p/ usuário }\end{array}$ \\
\hline $\begin{array}{l}\text { Gerente de } \\
\text { Células (GC) }\end{array}$ & $\begin{array}{l}\text { Orientado } \\
\text { a Objetivos }\end{array}$ & 1 & $\begin{array}{l}\text { Instancia a qdade de células para simulação } \\
\text { Recebe ordens do GGRID, replica para células } \\
\text { Recebe os estados das células, replica para GGRID }\end{array}$ \\
\hline $\begin{array}{l}\text { Gerente } \\
\text { Genérico (GG) }\end{array}$ & $\begin{array}{l}\text { Orientado } \\
\text { a Objetivos }\end{array}$ & 1 & $\begin{array}{l}\text { Instancia a qdade de GG necessários para a simulação } \\
\text { Recebe ordens do GGRID e replica para as células } \\
\text { Recebe os estados dos exploradores, replica para GGRID }\end{array}$ \\
\hline Célula & Reflexivo & $\begin{array}{l}\text { Definido pelo } \\
\text { usuário }\end{array}$ & $\begin{array}{l}\text { Recebe ordens do GC } \\
\text { Envia seu estado para o GC }\end{array}$ \\
\hline Explorador & Reflexivo & $\begin{array}{l}\text { Definido pelo } \\
\text { usuário }\end{array}$ & $\begin{array}{l}\text { Recebe ordens do GG } \\
\text { Move-se de uma célula para outra } \\
\text { Envia seu estado e posição para o GC }\end{array}$ \\
\hline
\end{tabular}

as quais podem ser expandidas ou criadas novas instâncias, à medida que os diferentes comportamentos dos agentes forem modelados. Atreladas à cada uma das classes de agentes estão imagens com o domínio espacial nativos à classe, como por exemplo, o mapa das áreas urbanas associada aos agentes urbanos, formando assim uma layer de simulação.

Para administrar a coordenação dos agentes, a cooperação e a resolução de conflitos, optou-se por uma organização em níveis hierárquicos. Nessa hierarquia, agentes de níveis mais altos possuem maior controle sobre agentes em níveis inferiores, conforme apresentado na Figura 10.

Na camada de usuário projetou-se uma interface que permitisse a configuração do modelo e obtenção dos resultados de simulação. Toda a configuração fornecida pelo usuário da ferramenta é traduzida em arquivos XML e carregada pelo protótipo de forma a iniciar os agentes e associá-los à camada de simulação.

O usuário configura quais agentes farão parte da simulação, adicionando comportamentos a eles a partir de uma biblioteca disponível. Também é possível associar 


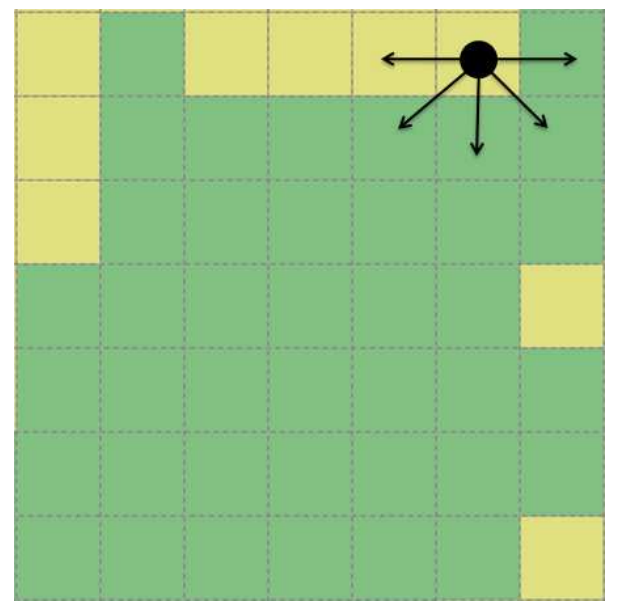

(a)

\begin{tabular}{|c|c|c|c|c|c|c|}
\hline 1 & 1 & 1 & 1 & 0 & & $\longrightarrow$ \\
\hline 1 & 1 & 1 & 1 & 1 & 1 & 0 \\
\hline 1 & 1 & 1 & 1 & 0 & 0 & 1 \\
\hline 1 & 0 & 0 & 0 & 0 & 1 & 1 \\
\hline 1 & 0 & 1 & 1 & 1 & 1 & 1 \\
\hline 0 & 0 & 1 & 1 & 1 & 1 & 1 \\
\hline 0 & 1 & 1 & 1 & 1 & 1 & 1 \\
\hline
\end{tabular}

(b)

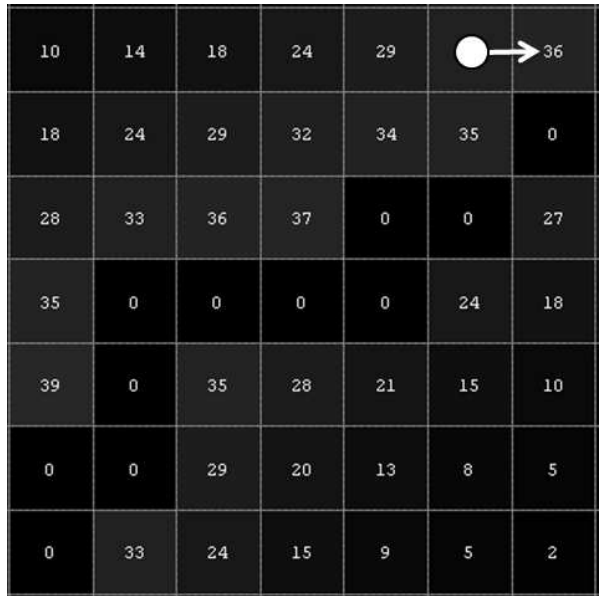

(c)

Figura 8. llustração do processo de deslocamento de um agente explorador a partir do mapa de uso antrópico, mostrado em (a), e dos mapas $T_{a u x}$ e $T$, mostados em (b) e (c), respectivamente.

máquinas de estados à cada agente, a fim de especificar o ciclo de vida de cada um. Além disso, regras adicionais, como diretrizes globais à simulação, são também colocadas nessa etapa. A flexibilidade da configuração estende-se à criação do número de agentes de cada classe, que interagirão no espaço definido pelo usuário por layers de simulação respectivas de cada classe.

A camada de controle se utiliza das regras e configurações definidas previamente para produzir a simulação. É nessa camada que estão os mecanismos de simulação, de sobreposição das layers e de armazenamento dos resultados intermediários. A camada física é responsável por carregar as imagens reais e compará-las com as imagens provenientes da simulação, calculando o PSNR (peak signal-to-noise ratio) entre as duas.

\section{Conclusão e Trabalhos Futuros}

O resultado esperado do trabalho em curso é a implementação e validação da ferramenta computacional de simulação, por meio de uma arquitetura baseada em SMA, para ana- 


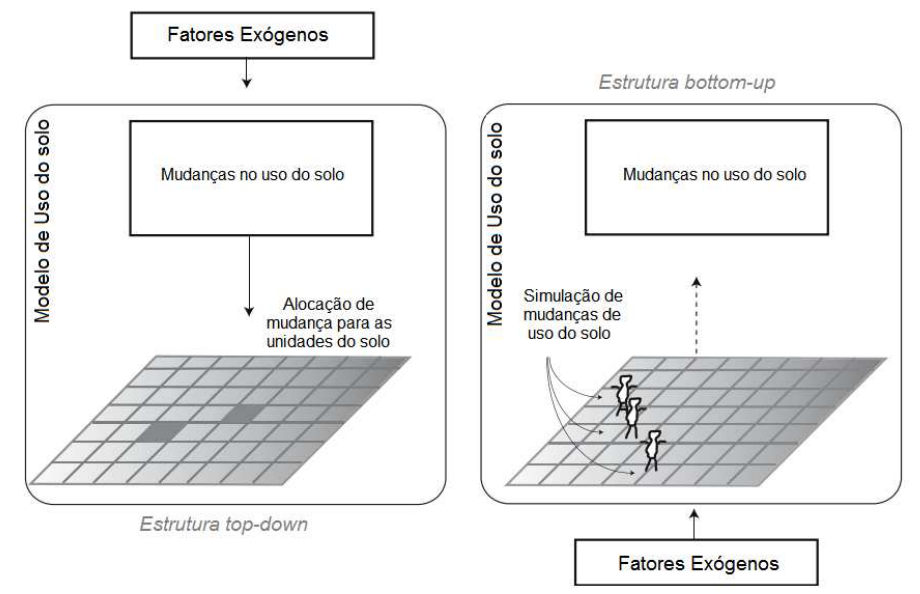

Figura 9. Representação da hierarquia na estrutura dos modelos de uso do solo (adaptado de Verburg, 2006).

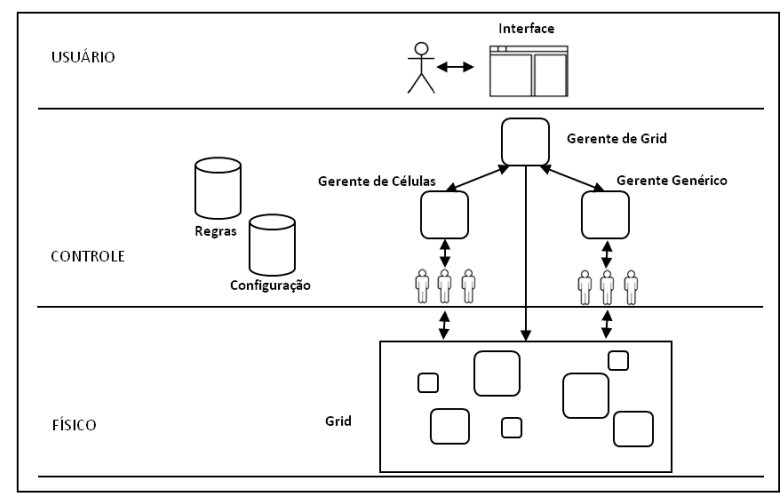

Figura 10. Proposta de Arquitetura em Níveis.

lisar a dinâmica de uso e cobertura do solo. Uma vez operacional, a ferramenta terá a finalidade de explorar cenários alternativos e investigar hipóteses para subsidiar propostas de políticas públicas de gestão ambiental. Os estudos realizados até o momento apontam SMA como uma iniciativa emergente e promissora, destacando o papel chave da Ciência da Computação para o fortalecimento da interdisciplinaridade com as Ciências Ecológicas.

É importante ressaltar que, com base nos estudos efetuados, não existe hoje ferramenta de abordagem SMA que apresente flexibilidade de representação espaço-temporal capaz de simular diferentes modelos de forma genérica. Ferramentas baseadas em outras técnicas que apresentam essa generalidade possuem uma série de restrições quanto à representação espacial (consideram o espaço homogêneo), quanto à dinamicidade (apresentam vizinhanças estacionárias) ou quanto à representação das tomadas de decisões tipicamente humanas (utilizam regras de transição universais). Este projeto explora essas lacunas, ao combinar a flexibilidade dos agentes com cálculos de mapas de influência para determinar comportamentos distintos e influência das adjacências, além de ser espacialmente explícito, simulando as interações em mapas reais de forma não homogênea.

A continuidade da pesquisa deverá explorar as características dos agentes e da plataforma, bem como a caracterização detalhada do modelo. Buscar-se-á o desenvolvi- 
mento de uma arquitetura flexível para a definição de agentes para que a interação entre as pressões naturais e antrópicas agindo no espaço possam ser reproduzidas.

Uma ferramenta de simulação baseada em agentes contribuirá para a área de IA e SMA por reproduzir o conhecimento e o raciocínio de agentes heterogêneos, coordenados de forma conjunta para que problemas de gestão sejam resolvidos. A área das Ciências Ecológicas também poderá explorar a dinamicidade que advém da abordagem de SMA para representar os problemas de complexidade, inerentes ao meio ambiente. Pela análise da dinâmica de uso e de ocupação do solo para o contexto proposto, será possível investigar a implicação de diferentes estratégias de conservação, gestão e/ou planejamento.

\section{Referências}

Agarwal, C., Green, G. M., Grove, J. M., Evans, T. P., and Schweik, C. M. (2001). A review and assessment of land-use change models: dynamics of space, time, and human choice. United States Department of Agriculture - USDA.

Bellifemine, F., Caire, G., Poggi, A., and Rimassa, G. (2008). Jade: A software framework for developing multi-agent applications. In Software: Practice and Experience, number 50, pages 10-21.

Bousquet, F., Bakam, I., Proton, H., and Page, C. L. (1998). Cormas: Common-Pool Resources and Multi-agent Systems. In IEA/AIE '98, pages 826-837.

Eastman, J. R. (2001). IDRISI 32.2 - guide to GIS and image processing. Clark Labs, Clark University, Worcester, USA.

Klink, C. A. and Machado, R. B. (2005). A conservação do Cerrado brasileiro. In Megabiodiversidade, volume 1, pages 147-155.

Marinelli, C. E., Coelho, C. G. C., Ralha, C. G., Zaghetto, A., and Macchiavello, B. (2010). Modelo de simulaçãao com uso de abordagem de sma para o zoneamento de unidades de conservação da amazônia. In VI Simpósio Brasileiro de Sistemas de Informação.

Sano, E. E., Rosa, R., Brito, J. L. S., and Ferreira, L. G. a. (2008). Mapeamento semidetalhado do uso da terra do bioma cerrado. In Pesquisa Agropecuária Brasileira, volume 43, pages 153-156.

Soares-Filho, B. S., Cerqueira, G. C., and Pennachin, C. L. (2002). DINAMICA: a stochastic cellular automata model designed to simulate the landscape dynamics in an Amazonian colonization frontier. In Ecological Modelling, volume 154.

Verburg, P. H. (2006). Simulating feedbacks in land use and land cover change models. In Landscape Ecology, volume 21, pages 1171-1183.

Verburg, P. H., Soepboer, W., Veldkamp, A., Limpiada, R., Espaldon, V., and Mastura, S. S. A. (2002). Modeling the spatial dynamics of regional land use: the CLUE-S model. In Environmental Management, volume 30, pages 391-405.

Weiss, G. (2000). Multiagent Systems: a Modern Approach to Distributed Artificial Intelligence. The MIT Press.

Wooldridge, M. (2009). An Introduction to Multiagent Systems. John Wiley \& Sons, 2nd edition. 